\title{
COMPOSITION OF BLOCHS WITH BOUNDED ANALYTIC FUNCTIONS
}

\author{
E. G. KWON
}

(Communicated by Theodore Gamelin )

ABSTRACT. If $f$ is a holomorphic self-map of the open unit disc and $1 \leq p<\infty$, then the following are equivalent.

(1) $h \circ f \in H^{2 p}$ for all Bloch functions $h$.

$$
\begin{gathered}
\sup _{r} \int_{0}^{2 \pi}\left(\log \frac{1}{1-\left|f\left(r e^{i \theta}\right)\right|^{2}}\right)^{p} d \theta<\infty . \\
\int_{0}^{2 \pi}\left(\int_{0}^{1}\left(f^{\#}\right)^{2}\left(r e^{i \theta}\right)(1-r) d r\right)^{p} d \theta<\infty,
\end{gathered}
$$

where $f^{\#}$ is the hyperbolic derivative of $f: f^{\#}=\left|f^{\prime}\right| /\left(1-|f|^{2}\right)$.

\section{INTRODUCTION}

Initiated by works of P. Ahern and W. Rudin ([A], $[\mathrm{AR}])$, there is extensive research on Bloch-to-BMOA pullbacks, that is, research on those holomorphic maps $f$ of the unit ball of $\mathbf{C}^{n}$ into the unit disc of $\mathbf{C}$ for which the composition operator defined by

$$
\mathcal{C}_{f}(h)=h \circ f
$$

takes Bloch functions to functions of BMOA. We refer to [RU] for recent research on Bloch to BMOA pullbacks.

It is known (see [RU], Section 5), when $n=1$, that one of the necessary and sufficient conditions for $\mathcal{C}_{f}$ to take all Blochs to BMOA is that $f$ be a function of $\mathrm{BMOA}_{\sigma}$, the Yamashita hyperbolic BMOA class (see [G] and [Y3] for BMOA and $\left.\mathrm{BMOA}_{\sigma}\right)$. This note is to resolve a natural parallelism to the phenomenon.

Theorem 1 (Main Result). If $f$ is a holomorphic self-map of the open unit disc and $1 \leq p<\infty$, then the following are equivalent.

(1) $\mathcal{C}_{f}$ takes Blochs to $H^{2 p}$, that is,

$$
h \circ f \in H^{2 p} \quad \text { for all Bloch functions } h .
$$

Received by the editors January 31, 1994 and, in revised form, October 19, 1994.

1991 Mathematics Subject Classification. Primary 30D55, 30D45.

Key words and phrases. $H^{p}$ space, Bloch space, hyperbolic Hardy class, pullbacks.

This paper was supported by NON DIRECTED RESEARCH FUND, Korea Research Foundation, 1993

(C)1996 American Mathematical Society 
(2) $f$ belongs to Yamashita's hyperbolic Hardy class $H_{\sigma}^{p}$, that is,

$$
\begin{aligned}
& \sup _{r} \int_{0}^{2 \pi}\left(\log \frac{1}{1-\left|f\left(r e^{i \theta}\right)\right|^{2}}\right)^{p} d \theta<\infty \\
& \int_{0}^{2 \pi}\left(\int_{0}^{1}\left(f^{\#}\right)^{2}\left(r e^{i \theta}\right)(1-r) d r\right)^{p} d \theta<\infty,
\end{aligned}
$$

where $f^{\#}$ is the hyperbolic derivative of $f: f^{\#}=\left|f^{\prime}\right| /\left(1-|f|^{2}\right)$.

\section{Preliminaries}

The Bloch space $\mathcal{B}$ consists of those $f$ holomorphic in the open unit disc $D$ of the complex plane for which

$$
\|f\|_{\mathcal{B}}:=\sup _{z \in D}\left|f^{\prime}(z)\right|\left(1-|z|^{2}\right)<\infty .
$$

We let $1 \leq p<\infty$ and set for $f$ subharmonic in $D$

$$
\|f\|_{p}:=\sup _{r}\left(\int_{0}^{2 \pi}\left|f\left(r e^{i \theta}\right)\right|^{p} \frac{d \theta}{2 \pi}\right)^{1 / p} .
$$

Then $H^{p}=H^{p}(D)$ consists of those $f$ holomorphic in $D$ for which $\|f\|_{p}<\infty$. See [D] and [G] for Bloch and $H^{p}$ spaces.

The Yamashita hyperbolic Hardy class $H_{\sigma}^{p}$ is defined as the set of those holomorphic self-maps $f$ of $D$ for which

$$
\|\sigma(f)\|_{p}<\infty,
$$

where $\sigma(z)$ denotes the hyperbolic distance of $z$ and 0 in $D$, namely,

$$
\sigma(z)=\frac{1}{2} \log \frac{1+|z|}{1-|z|}
$$

Though $H_{\sigma}^{p}$ is not a linear space, it has, as hyperbolic counterparts, many properties analogous to those of $H^{p}$. We let $T$ be the boundary of $D$ and set, following Yamashita,

$$
\lambda(f)=\log \frac{1}{1-|f|^{2}} \quad \text { and } \quad f^{\#}=\frac{\left|f^{\prime}\right|}{1-|f|^{2}}
$$

for the holomorphic self-map $f$ of $D$. Then $\sigma(f)^{p}, \lambda(f)^{p}$, and $\left(f^{\#}\right)^{p}$ are subharmonic functions, so that their integral means over $r T$ are nondecreasing functions of $r$ : for example,

$$
\int_{0}^{2 \pi} \lambda(f)^{p}\left(r e^{i \theta}\right) \frac{d \theta}{2 \pi} \nearrow\|\lambda(f)\|_{p}^{p} \text { as } \quad r \nearrow 1 .
$$

Also, there are corresponding maximal theorems for these functions: Set

$$
M_{\lambda}(f, \theta)=\sup \left\{\lambda(f)\left(r e^{i \theta}\right): 0 \leq r<1\right\} ;
$$


then

$$
\left\|M_{\lambda}(f, .)\right\|_{L^{p}} \leq C_{p}\|\lambda(f)\|_{p}
$$

for $f \in H_{\sigma}^{p}$ ([Y1]). The left side of $(2.1)$ means usual $L^{p}(T)$ norm. The function $f^{\#}$ is the hyperbolic counterpart of $f^{\prime}$ and it easily follows that

$$
\frac{1}{2} \lambda(f) \leq \sigma(f)<\frac{1}{2} \lambda(f)+\log 2
$$

and

$$
\Delta\left(\lambda(f)^{p}\right)=4 p\left\{(p-1)|f|^{2}+\lambda(f)\right\} \lambda(f)^{p-2}\left(f^{\#}\right)^{2},
$$

where $\Delta$ denotes the Laplacian:

$$
\Delta=4 \frac{\partial^{2}}{\partial z \partial \bar{z}}
$$

From (2.2) and (2.3), it should be noted that

$$
f \in H_{\sigma}^{p} \quad \text { if and only if } \quad\|\lambda(f)\|_{p}<\infty
$$

and

$$
\Delta\left(\lambda(f)^{p}\right) \sim \lambda(f)^{p-1}\left(f^{\#}\right)^{2} .
$$

Here and after $\psi \sim \phi$ means that either both sides are zero or the quotient $\psi / \phi$ lies between two positive uniform constants. See, for example, [K1], [K2], [Y1], and [Y2] for the theory of $H_{\sigma}^{p}$.

In the remaining sections, we confine $f$ to being a holomorphic self-map of $D$ and denote $f_{r}, 0 \leq r \leq 1$, as the function defined by $f_{r}(z)=f(r z), z \in D$. Positive constants depending on $p$ (or $q$ ) will be denoted by $C_{p}$ (or $C_{q}$ ), whose quantities may vary at each occurrence.

\section{Hyperbolic $g$-FUnCTION}

For $h$ holomorphic in $D, g$-function of Paley defined by

$$
g(\theta):=g(h)(\theta)=\left(\int_{0}^{1}\left|h^{\prime}\right|^{2}\left(r e^{i \theta}\right)(1-r) d r\right)^{1 / 2}, \quad 0 \leq \theta<2 \pi,
$$

satisfies $\|g(h)\|_{L^{p}} \sim\|h\|_{p}$ if $h(0)=0([\mathrm{Z}])$. Consider Green's theorem of the form

$$
r \int_{0}^{2 \pi} \frac{\partial \psi}{\partial r} d \theta=\iint_{|z| \leq r} \Delta \psi d x d y,
$$

valid for $\psi \in C^{2}(D)$. If we integrate both sides with respect to $d r$ after dividing them by $r$ and applying $\psi=\lambda(f)^{p}$, then we obtain, by use of (2.4),

$$
\begin{aligned}
& \left\|\lambda\left(f_{\rho}\right)\right\|_{p}^{p}-\lambda(f)^{p}(0) \\
\sim & \frac{1}{2 \pi} \int_{0}^{\rho} \frac{d r}{r} \int_{|z| \leq r} \int_{\mid \leq r} \lambda(f)^{p-1}\left(f^{\#}\right)^{2}(z) d x d y \\
= & \frac{1}{2 \pi} \iint_{|z|<\rho} \lambda(f)^{p-1}\left(f^{\#}\right)^{2}(z) \log \frac{\rho}{|z|} d x d y, \quad 0 \leq \rho \leq 1 .
\end{aligned}
$$


In particular, we see from (3.2) that $f \in H_{\sigma}^{1}$ if and only if

$$
\infty>\iint_{D}\left(f^{\#}\right)^{2}(z) \log \frac{1}{|z|} d x d y
$$

This suggests we define the hyperbolic version of $g$-function using $f^{\#}$. We define

$$
g_{\sigma}(\theta):=g_{\sigma}(f)(\theta)=\int_{0}^{1}\left(f^{\#}\right)^{2}\left(r e^{i \theta}\right)(1-r) d r, 0 \leq \theta<2 \pi .
$$

It is not surprising to see the absence of the square root in the definition of $g_{\sigma}$ in (3.3) when we compare it to that of $g$ - function in (3.1), because there is a known parallelism (see [Y2]) between $H^{2}$ and $H_{\sigma}^{1}$.

Theorem 2. If $1 \leq p<\infty$, then the following are equivalent.

(1) $f \in H_{\sigma}^{p}$.

(2) $g_{\sigma}(f) \in L^{p}(T)$.

In fact, $\|\lambda(f)\|_{p} \sim\left\|g_{\sigma}(f)\right\|_{L^{p}}$ provided $f(0)=0$.

Proof. By (3.2) and (3.3), there is nothing to prove when $p=1$. We assume $1<p<\infty$, and let $\frac{1}{p}+\frac{1}{q}=1$.

$(1) \Longrightarrow(2)$ We begin with the identity

$$
\left\|g_{\sigma}\right\|_{L^{p}}=\sup \int_{0}^{2 \pi} g_{\sigma}(\theta) h(\theta) \frac{d \theta}{2 \pi}
$$

where the supremum is taken with respect to all nonnegative trigonometric polynomials $h$ with $\|h\|_{L^{q}} \leq 1$. Since $\left(f^{\#}\right)^{2}$ is subharmonic, we have

$$
\left(f^{\#}\right)^{2}\left(r^{2} e^{i \theta}\right) \leq \int_{0}^{2 \pi} P(r, \theta-t)\left(f^{\#}\right)^{2}\left(r e^{i t}\right) \frac{d t}{2 \pi}, \quad 0 \leq r<1,
$$

where $P(r, \theta)$ is the Poisson kernel:

$$
P(r, \theta)=\frac{1-r^{2}}{1-2 r \cos \theta+r^{2}} .
$$

Let $u$ be the Poisson integral of $h$. Then

$$
\begin{aligned}
& \int_{0}^{2 \pi} g_{\sigma}(\theta) h(\theta) d \theta \\
= & \int_{0}^{2 \pi} \int_{0}^{1}\left(f^{\#}\right)^{2}\left(r e^{i \theta}\right) h(\theta)(1-r) d r d \theta \\
= & \int_{0}^{2 \pi} \int_{0}^{1}\left(f^{\#}\right)^{2}\left(r^{2} e^{i \theta}\right) h(\theta)\left(1-r^{2}\right) 2 r d r d \theta \\
\leq & 2 \iint_{D}\left(f^{\#}\right)^{2}(z) u(z)\left(1-|z|^{2}\right) d x d y \\
\leq & 4 \iint_{D}\left(f^{\#}\right)^{2}(z) u(z) \log \frac{1}{|z|} d x d y,
\end{aligned}
$$


where we changed the order of integration and used (3.4) in the first inequality.

If we denote $\partial=\frac{1}{2}\left(\frac{\partial}{\partial x}-i \frac{\partial}{\partial y}\right)$ and $\bar{\partial}=\frac{1}{2}\left(\frac{\partial}{\partial x}+i \frac{\partial}{\partial y}\right), z=x+i y$, then it follows from elementary calculation and (2.3) that

$$
4\left(f^{\#}\right)^{2} u=\Delta(\lambda(f) u)-4(\partial \lambda(f) \bar{\partial} u+\bar{\partial} \lambda(f) \partial u),
$$

so that by (3.5) we have

$$
\begin{aligned}
& \int_{0}^{2 \pi} g_{\sigma}(\theta) h(\theta) d \theta \\
\leq & \left|\iint_{D} \Delta(\lambda(f) u)(z) \log \frac{1}{|z|} d x d y\right| \\
& +4 \iint_{D}|\partial \lambda(f) \bar{\partial} u+\bar{\partial} \lambda(f) \partial u|(z) \log \frac{1}{|z|} d x d y \\
= & (I)+(I I) .
\end{aligned}
$$

Now, using Green's theorem (as in $(3.2)$ with $p=1$ ) with limiting process and Hölder's inequality, we obtain

$$
\begin{aligned}
(I) & =\left|\lim _{\rho \rightarrow 1} \int_{0}^{2 \pi}(\lambda(f) u)\left(\rho e^{i \theta}\right) d \theta-2 \pi(\lambda(f) u)(0)\right| \\
& \leq 2 \pi\|\lambda(f)\|_{p}\|u\|_{q} \leq 2 \pi\|\lambda(f)\|_{p} .
\end{aligned}
$$

On the other hand, if we let $\phi$ be a holomorphic function in $D$ whose real part is $u$, it then follows from direct differentiation that

$$
|\partial \lambda(f)|=|\bar{\partial} \lambda(f)|=|f| f^{\#}
$$

and

$$
|\partial u|=|\bar{\partial} u|=\frac{1}{2}\left|\phi^{\prime}\right|
$$

Hence

$$
\begin{aligned}
(I I) & \leq 4 \iint_{D}\left|\phi^{\prime}(z)\right||f(z)| f^{\#}(z) \log \frac{1}{|z|} d x d y \\
& \leq 4 \int_{0}^{2 \pi} \int_{0}^{1}\left|\phi^{\prime}\left(r e^{i \theta}\right)\right|\left|f\left(r e^{i \theta}\right)\right| f^{\#}\left(r e^{i \theta}\right)(1-r) d r d \theta .
\end{aligned}
$$

Since $\left|f\left(r e^{i \theta}\right)\right| \leq \sqrt{\lambda(f)\left(r e^{i \theta}\right)} \leq M_{\lambda}^{1 / 2}(f, \theta)$, we have, by the Schwarz inequality,

$$
(I I) \leq 4 \int_{0}^{2 \pi} M_{\lambda}^{1 / 2}(f, \theta) \sqrt{g_{\sigma}(\theta)} g_{\phi}(\theta) d \theta,
$$

where $g_{\phi}(\theta)$ is Paley $g$-function of $\phi$. Applying Hölder's inequality with indices $2 p$, $2 p, q$ to the right side of (3.8) and using maximal theorem (2.1), we arrive at

$$
(I I) \leq C_{p}\|\lambda(f)\|_{p}^{1 / 2}\left\|g_{\sigma}(f)\right\|_{L^{p}}^{1 / 2}\left\|g_{\phi}\right\|_{L^{q}}
$$


From the theory of $g$-function, we know $\left\|g_{\phi}\right\|_{L^{q}} \leq C_{q}\|\phi\|_{q}$, and it follows from the theorem of M. Riesz ([Z]) that $\|\phi\|_{q} \leq C_{q}\|u\|_{q} \leq C_{q}$. Thus

$$
(I I) \leq C_{p}\|\lambda(f)\|_{p}^{1 / 2}\left\|g_{\sigma}(f)\right\|_{L^{p}}^{1 / 2} .
$$

Hence, combining estimates (3.6), (3.7), and (3.9), we have

$$
\begin{aligned}
\int_{0}^{2 \pi} g_{\sigma}(\theta) h(\theta) d \theta & \leq(I)+(I I) \\
& \leq 2 \pi\|\lambda(f)\|_{p}+C_{p}\|\lambda(f)\|_{p}^{1 / 2}\left\|g_{\sigma}(f)\right\|_{L^{p}}^{1 / 2},
\end{aligned}
$$

for all positive trigonometric polynomials $h$ with $\|h\|_{q} \leq 1$. Therefore we obtain

$$
\left\|g_{\sigma}(f)\right\|_{L^{p}} \leq\|\lambda(f)\|_{p}+C_{p}\|\lambda(f)\|_{p}^{1 / 2}\left\|g_{\sigma}(f)\right\|_{L^{p}}^{1 / 2}, \quad f \in H_{\sigma}^{p} .
$$

Now we conclude from (3.10) that

$$
\left\|g_{\sigma}(f)\right\|_{L^{p}} \leq C_{p}\|\lambda(f)\|_{p}
$$

In fact, if $f \equiv 0$, then there is nothing to prove; otherwise, setting $X(r)=X(f, p, r)$ $=\left(\left\|g_{\sigma}\left(f_{r}\right)\right\|_{L^{p}} /\left\|\lambda\left(f_{r}\right)\right\|_{p}\right)^{\frac{1}{2}}, 0<r<1,(3.10)$ with $f_{r}$ in place of $f$ becomes

$$
X^{2}(r) \leq 1+C_{p} X(r)
$$

and this means, by comparing the order of $X(r)$, that $X(r), 0<r<1$, does not exceed the larger root of the equation $X^{2}=1+C_{p} X$. This proves (3.11) with $f_{r}$, $0 \leq r<1$, in place of $f$, and so (3.11) follows by the monotonicity of both sides.

$(2) \Longrightarrow$ (1) It follows from (3.2) that

$$
\begin{aligned}
\left\|\lambda\left(f_{r}\right)\right\|_{p}^{p}-\lambda(f)^{p}(0) & \sim C_{p} \int_{0}^{2 \pi} \frac{d \theta}{2 \pi} \int_{0}^{r}\left(f^{\#}\right)^{2} \lambda(f)^{p-1}\left(\rho e^{i \theta}\right) \log \frac{r}{\rho} \rho d \rho \\
& \leq C_{p} \int_{0}^{2 \pi} M_{\lambda}^{p-1}\left(f_{r}, \theta\right) g_{\sigma}(f)(\theta) \frac{d \theta}{2 \pi} .
\end{aligned}
$$

Hölder's inequality and the maximal theorem show the last quantity to be bounded by

$$
C_{p}\left\|\lambda\left(f_{r}\right)\right\|_{p}^{p-1}\left\|g_{\sigma}(f)\right\|_{L^{p}}
$$

so that we have

$$
\left\|\lambda\left(f_{r}\right)\right\|_{p}^{p}-\lambda(f)^{p}(0) \leq C_{p}\left\|\lambda\left(f_{r}\right)\right\|_{p}^{p-1}\left\|g_{\sigma}(f)\right\|_{L^{p}} .
$$

If $g_{\sigma} \equiv 0$, there remains nothing to prove. Otherwise, under the assumption $0<$ $\left\|g_{\sigma}\right\|_{L^{p}}<\infty$, by considering the order of

$$
Y(r)=Y(f, p, r)=\left(\left\|\lambda\left(f_{r}\right)\right\|_{p} /\left\|g_{\sigma}\left(f_{r}\right)\right\|_{L^{p}}\right), 0<r<1,
$$

we conclude that $Y(r), 0<r<1$, does not exceed the largest root of the equation

$$
Y^{p}-\frac{\lambda(f)^{p}(0)}{\left\|g_{\sigma}(f)\right\|_{L^{p}}^{p}}=C_{p} Y^{p-1}
$$

and from this follows

$$
\|\lambda(f)\|_{p} \leq C_{p}(f)\left\|g_{\sigma}(f)\right\|_{L^{p}}
$$

Here, $C_{p}(f)$ denotes a constant depending on $p$ and $f$.

The last assertion of Theorem 2 follows from (3.11) and (3.12). 


\section{BLOCH TO $H^{p}$ PULlbaCKS}

Now we prove our main theorem, Theorem 1. By the aid of Theorem 2, it suffices to prove the following.

Theorem 3. If $1 \leq p<\infty$, then the following are equivalent.

(1) $g_{\sigma}(f) \in L^{p}(T)$.

(2) $\mathcal{C}_{f}$ takes Blochs to $H^{2 p}$.

Proof. (1) $\Longrightarrow(2)$ Let $h \in \mathcal{B}$. Then

$$
\left|\left(\mathcal{C}_{f} h\right)^{\prime}\right|=\left|(h \circ f)^{\prime}\right| \leq\|h\|_{\mathcal{B}} f^{\#} .
$$

Hence

$$
\begin{aligned}
& \left\|g_{h \circ f}\right\|_{L^{2 p}}^{2 p} \\
= & \int_{0}^{2 \pi}\left(\int_{0}^{1}\left|(h \circ f)^{\prime}\left(r e^{i \theta}\right)\right|^{2}(1-r) d r\right)^{p} \frac{d \theta}{2 \pi} \\
\leq & \|h\|_{\mathcal{B}}^{2 p} \int_{0}^{2 \pi}\left(\int_{0}^{1}\left(f^{\#}\right)^{2}\left(r e^{i \theta}\right)(1-r) d r\right)^{p} \frac{d \theta}{2 \pi} \\
= & \|h\|_{\mathcal{B}}^{2 p}\left\|g_{\sigma}(f)\right\|_{L^{p}}^{p} .
\end{aligned}
$$

Therefore $\|h \circ f\|_{2 p}<\infty$ if $g_{\sigma}(f) \in L^{p}(T)$.

$(2) \Longrightarrow(1)$ Using $g$-function, (2) says that

$$
\int_{0}^{2 \pi}\left(\int_{0}^{1}\left|(h \circ f)^{\prime}\right|^{2}\left(r e^{i \theta}\right)(1-r) d r\right)^{p} d \theta<\infty \quad \text { if } \quad h \in \mathcal{B} .
$$

On the other hand, W. Ramey and D. Ullrich ([RU], Proposition 5.4) constructed two Bloch functions $h_{j}, j=1,2$, such that

$$
\left(1-|z|^{2}\right)\left(\left|h_{1}^{\prime}(z)\right|+\left|h_{2}^{\prime}(z)\right|\right) \geq 1, \quad z \in D .
$$

From (4.4) it follows that $\left(\left|h_{1}^{\prime} \circ f\right|+\left|h_{2}^{\prime} \circ f\right|\right) \geq\left(1-|f|^{2}\right)^{-1}$, so that

$$
\begin{aligned}
& \left(\int_{0}^{1}\left|\left(h_{1} \circ f\right)^{\prime}\right|^{2}(1-r) d r\right)^{p}+\left(\int_{0}^{1}\left|\left(h_{2} \circ f\right)^{\prime}\right|^{2}(1-r) d r\right)^{p} \\
\geq & 2^{-2 p}\left(\int_{0}^{1}\left|f^{\prime}\right|^{2}\left(\left|h_{1}^{\prime} \circ f\right|+\left|h_{2}^{\prime} \circ f\right|\right)^{2}(1-r) d r\right)^{p} \\
\geq & 2^{-2 p}\left(\int_{0}^{1} \frac{\left|f^{\prime}\right|^{2}}{\left(1-|f|^{2}\right)^{2}}(1-r) d r\right)^{p} \\
= & 2^{-2 p} g_{\sigma}^{p}(f) .
\end{aligned}
$$

Now, integrating (4.5) with respect to $d \theta$ and applying (4.3) with $h_{j}, j=1,2$, in place of $h$, we obtain

$$
\left\|g_{\sigma}(f)\right\|_{p} \leq C_{p}\left(\left\|h_{1} \circ f\right\|_{2 p}+\left\|h_{2} \circ f\right\|_{2 p}\right) .
$$

This completes the proof. 


\section{Extreme POINTS OF $H^{\infty}$}

$H^{\infty}$ denotes, as usual, the space of bounded holomorphic functions on $D$. A well-known theorem of deLeeuw and Rudin ([D], Theorem 7.9) says that

$$
\int_{0}^{2 \pi} \log \frac{1}{1-\left|f\left(e^{i \theta}\right)\right|^{2}} d \theta=\infty
$$

is necessary and sufficient for a holomorphic $f$ with $\|f\|_{\infty}=\sup _{z \in D}|f(z)|=1$ to be an extreme point of the closed unit ball of $H^{\infty}$. The following is a direct corollary of Theorem 1.

Corollary 4. Let $f \in H^{\infty},\|f\|_{\infty}=1$. Then the following are equivalent.

(1) $f$ is an extreme point of the closed unit ball of $H^{\infty}$.

(2) $h \circ f \notin H^{2}$ for some $h \in \mathcal{B}$.

\section{ACKNOWLEDGMENT}

The author wishes to express his sincere gratitude to the referee for his helpful suggestion.

\section{REFERENCES}

[A] Patrick R. Ahern, On the behavior near torus of functions holomorphic in the ball, Pacific J. Math. 107 (1983), 267-278. MR 84i:32023

[AR] Patrick R. Ahern and Walter Rudin, Bloch functions, BMO, and boundary zeros, Indiana Univ. Math. J. 36 (1987), 131-148. MR 88d:42036

[D] Peter. L. Duren, The theory of $H^{p}$ functions, Academic Press, New York, 1970. MR 42:3552

[G] John. B. Garnett, Bounded analytic functions, Academic Press, New York, 1981. MR 83g:30037

[K1] E. G. Kwon, Fractional integration and the hyperbolic derivative, Bull. Austral. Math. Soc. 38 (1988), 357-364. MR 90a:30096

[K2] - Mean growth of the hyperbolic Hardy class functions, Math. Japonica 35 (1990), 451-460. MR 91e:30064

[RU] Wade Ramey and David Ullrich, Bounded mean oscillations of Bloch pullbacks, Math. Ann. 291 (1991), 591-606. MR 92i:32004

[Y1] Shinji Yamashita, Functions with $H^{p}$ hyperbolic derivative, Math. Scand. 13 (1983), 238244. MR 85f:30055

[Y2] - Hyperbolic Hardy classes and hyperbolically Dirichlet finite functions, Hokkaido Math. J. 10 (1981), 709-722, Special Issue.

[Y3] - Holomorphic functions of hyperbolically bounded mean oscillations, Bollentino U.M.I. 5-B (1986), no. 6, 983-1000. MR 88e:30092

[Z] A. Zygmund, Trigonometric series, Cambridge Univ. Press, London, 1959. MR 21:6498

Department of Mathematics-Educhtion, Andong National University, Andong 760-749, KOREA

E-mail address: egkwon@anu.andong.ac.kr 